\title{
Prendre place : les Boliviens sur les marchés itinérants de Buenos Aires
}

Taking Place: Bolivians in Buenos Aires Itinerant Street Markets

Tomar sitio: los bolivianos en las ferias itinerantes de Buenos Aires

Laurence Brassamin et Virginie Baby-Collin

\section{(2) OpenEdition}

Édition électronique

URL : https://journals.openedition.org/remi/6098

DOI : 10.4000/remi.6098

ISSN : $1777-5418$

Éditeur

Université de Poitiers

Édition imprimée

Date de publication : 1 septembre 2012

Pagination : 127-149

ISBN : 979-10-90426-05-4

ISSN : 0765-0752

\section{Référence électronique}

Laurence Brassamin et Virginie Baby-Collin, «Prendre place : les Boliviens sur les marchés itinérants de Buenos Aires », Revue européenne des migrations internationales [En ligne], vol. 28 - n³ | 2012, mis en ligne le 01 septembre 2015, consulté le 14 avril 2022. URL : http://journals.openedition.org/remi/ 6098 ; DOI : https://doi.org/10.4000/remi.6098 


\section{Prendre place : \\ les Boliviens sur les marchés itinérants de Buenos Aires}

\section{Laurence Brassamin ${ }^{1}$ et Virginie Baby-Collin²}

Raul, Bolivien âgé de quarante-huit ans en 2009, originaire de Cochabamba, est arrivé en 1977 à Buenos Aires, à l'âge de vingt-six ans. Les premiers jours, dans la grande gare de Retiro, il propose ses services pour charger et décharger les marchandises. II y rencontre un compadre bolivien, en l'occurrence un cousin, qui l'héberge pendant trois ans à la Matanza, une municipalité de la couronne périphérique de Buenos Aires où vit une importante population bolivienne. Ce compadre travaillant dans la construction, il lui propose de rejoindre son équipe où Raul reste sept ans. Alors que les Boliviens deviennent d'importants acteurs de l'agriculture périurbaine, notamment à La Matanza, Raul tente de constituer une exploitation agricole entre 1984 et 1987. Il échoue et s'oriente vers la vente dans l'un des marchés de gros de fruits et légumes de la périphérie, puis vers la vente informelle dans les marchés de rue de la capitale. Il se spécialise dans les produits fermiers (poulets, œufs), puis obtient un premier permis officiel de travail en 2002 (vingt-cinq ans après son arrivée), pour un stand sur la FIAB3 $n^{\circ} X 4$, dont il lègue le titre à son fils, puis deux autres permis en 2003 sur les FIAB $n^{\circ} X$ et $Y$, qu'il partage avec sa femme. Il est aujourd'hui propriétaire d'une maison dans le quartier périphérique de Villa Celina, à la lisière de la ville. Lorsqu'on le croise sur les marchés, il a des allures de chef d'entreprise dynamique, avec ses deux portables qui sonnent régulièrement, ses va-et-vient pour gérer les marchandises, les contacts, les affaires des trois stands familiaux.

Cette success-story illustre l'insertion professionnelle et urbaine des Boliviens dans les espaces commerciaux de Buenos Aires. En Amérique latine, le commerce occupe une place importante dans l'espace public et les marchés sont des lieux de référence et d'échanges économiques, sociaux et culturels. Espaces de prédilection de l'économie de survie et du commerce informel, les ferias ont connu un véritable boom lors de la crise argentine de 2001, via de nombreuses initiatives populaires réinventant le troc sur les marchés (Busso, 2007).

\footnotetext{
1 Géographe et Journaliste, Table de Concertation des organismes au service des personnes Immigrantes et Réfugiées (TCRI), 518 rue Beaubien Est, Montréal Qc Canada H2S 1S5 ;

I.brassamin@gmail.com

2 Maître de conférences, Géographe, Université d'Aix Marseille, UMRTelemme, MMSH, Université d'Aix Marseille, Département géographie, 29 avenue Robert Schuman, 13621 Aix-en-Provence Cedex ; virginie.baby-collin@univ-amu.fr

3 Feria itinerante de abastecimiento barrial, ou marché itinérant de quartier, réglementé par la mairie de Buenos Aires.

4 Les noms des personnes interrogées ainsi que les numéros des ferias ont été modifiés pour des raisons de confidentialité.
} 
La région métropolitaine de Buenos Aires, qui regroupe le tiers de la population argentine ${ }^{5}$, est le principal pôle de concentration de l'immigration étrangère (Carte 1). Les Boliviens sont le second groupe étranger au niveau national ( $19 \%$ des étrangers) derrière les Paraguayens (30\% des étrangers) et plus de la moitié d'entre eux (55\%) vit dans le Grand Buenos Aires ${ }^{6}$. Parmi les secteurs d'activité économique investis par les Boliviens on peut citer, outre les emplois classiques dans la construction, des ouvriers dans des ateliers textiles et dans d'autres industries de main-d'œuvre, dans le secteur agricole, notamment maraîcher en contexte périurbain, et dans les services domestiques. L'activité commerciale est de même très représentée, a fortiori en milieu urbain ${ }^{7}$. Outre la visibilité de la "Bolivienne du coin de la rue ", figure emblématique de la vendeuse informelle à proximité des supermarchés de la capitale qui vend des produits frais, souvent des fruits et légumes, la présence bolivienne est lisible dans les activités commerciales de rue, sur les marchés informels et dans les espaces de vente de rue formalisés.

Notre article porte sur les espaces formalisés de vente, rue et marchés itinérants, créés dans la ville autonome de Buenos Aires, gérés et légiférés par le gouvernement municipal. Nous cherchons à comprendre le fonctionnement des acteurs commerçants et à décrypter comment, à partir du début des années 1990, les Boliviens, très présents dans le commerce informel de rue, prennent place dans cette économie marchande formelle, alors que leur présence dans la capitale se consolide depuis les années 1960. Quelles trajectoires migratoires, sociales et professionnelles, permettent cette présence croissante des Boliviens sur les marchés ? Dans quelle mesure le contexte économique et politique local a-t-il joué un rôle dans l'accroissement de la visibilité commerciale bolivienne dans la capitale argentine ? Quel est le rôle des réseaux sociaux boliviens dans la construction de cette niche professionnelle ? Finalement, comment le rôle des Boliviens sur les marchés itinérants met-il en évidence leur capacité à prendre place dans la cité ?

\footnotetext{
5 Selon le recensement $2010: 40,117$ millions d'habitants en Argentine, dont 12,8 millions dans I'aire métropolitaine ou Grand Buenos Aires (2,89 millions dans la ville de Buenos Aires plus 9,9 millions dans les vingt-quatre municipalités de la périphérie), soit $31,92 \%$ de la population. Ce chiffre est légèrement accru si l'on considère la région métropolitaine de Buenos Aires (quarante municipalités) qui inclut seize municipalités supplémentaires de la grande couronne périphérique, inscrites dans le bassin de vie de la capitale par des mouvements pendulaires, et également récipiendaires de migrations étrangères (Carte 1).

6 Selon le recensement 2010, le pays compte 345272 Boliviens dont 76609 dans la ville autonome de Buenos Aires (où ils représentent $20 \%$ des étrangers) et 114146 dans les vingt-quatre municipalités périphériques du Grand Buenos Aires (soit 15,3\% des étrangers de la périphérie). Les 190755 Boliviens (17\% des étrangers) du Grand Buenos Aires représentent ainsi $55 \%$ des Boliviens officiellement recensés. Les chercheurs estiment à plus de 1 million les Boliviens en Argentine, dont la moitié dans la région de Buenos Aires (Sassone, 2002 et 2007). Le seul programme de régularisation des migrants clandestins du Mercosur entrés en Argentine avant 2006, appelé Patria Grande (2006-2009), a permis la régularisation de plus de 680000 migrants, dont plus de 202000 Boliviens (Novick, 2010). Les données de la carte 1 reprennent celles du recensement de 2001 : elles ne sont en effet pas encore disponibles à l'échelle municipale pour le recensement 2010.

7 Contrairement aux Paraguayens, très nombreux dans le service domestique, les Boliviens sont plus représentés dans le tertiaire non domestique et notamment dans le commerce ; les données de l'enquête ECMI, réalisée par I'INDEC en 2003, indiquent que pour la ville autonome de Buenos Aires, les Paraguayens travaillent à $40 \%$ dans le service domestique (contre $12 \%$ pour les Boliviens) et les Boliviens à $44 \%$ dans le secteur tertiaire non domestique ( $40 \%$ pour les Paraguayens); dans les municipalités du Grand Buenos Aires, les Boliviens sont représentés à $47 \%$ dans les activités tertiaires non domestiques, les Paraguayens à $34 \%$. Si I'on reste dans la ville autonome de Buenos Aires, le second secteur d'activité des Boliviens est le secondaire (31\%), puis la construction (13\%).
} 
Ce travail repose sur une enquête de terrain réalisée en 2009 et 2010 sur les marchés formels itinérants de la ville autonome de Buenos Aires - FIAB et FIIS (Ferias Itinerantes de Abasteciemiento Barrial et Ferias Itinerantes de Interés Social). Ces petites structures itinérantes sont spécialisées dans la vente de produits frais de consommation alimentaire courante (Brassamin, 2010). Nous avons recueilli une soixantaine d'entretiens sous forme de récits de vie auprès de vendeurs, majoritairement d'origine bolivienne, ainsi qu'une dizaine d'entretiens auprès d'acteurs institutionnels (Direction Générale du Commerce et du Marché, inspecteurs des ferias, vice-consul de Bolivie, représentants d'associations boliviennes à Buenos Aires) ${ }^{8}$.

Après avoir présenté le commerce de rue à Buenos Aires, les spécificités des marchés étudiés et leur fonctionnement entre formel et informel, nous interrogerons la présence bolivienne à partir des trajectoires des migrants et de la diversité de leurs statuts sur les marchés. Filières professionnelles, réseaux sociaux et liens locaux permettront de comprendre comment les Boliviens ont affirmé leur présence au cœur de la ville et non plus seulement sur ses franges informelles, profitant de conjonctures spécifiques.

\section{Les espaces marchands de rue à Buenos Aires. Entre formel et informel, des lieux d'importante présence bolivienne}

\section{Marchés et commerce de rue}

Le gouvernement de la ville autonome de Buenos Aires dispose d'une classification des marchés selon leurs activités, leur rythme d'activité et leur localisation : (1) cinq centres d'approvisionnement quotidiens (fixes) de grande taille et aux prix peu élevés, aux plafonds réglementés, qui proposent des biens de consommation élémentaires ; (2) un certain nombre de ferias, "passages commerciaux ", et un marché aux puces, qui se tiennent généralement du vendredi au dimanche, et sont spécialisés dans l'artisanat, les livres, les antiquités ; (3) un certain nombre de ferias itinérantes de vente au détail, FIAB et FIIS, objet de notre étude. La vente informelle (non classifiée par le gouvernement municipal) constitue enfin une part non négligeable de l'offre commerciale située dans l'espace public. Fort diversifiée, elle va des "Boliviennes du coin de la rue " et vendeurs à la sauvette aux grands marchés de rue quotidiens de certains quartiers (tels Liniers, cf. infra), en passant par les ferias hebdomadaires (fonctionnant généralement les fins de semaine) inégalement consolidées (Bonorino, cf. infra). À ces espaces commerciaux ouverts de vente au détail, en dehors des limites municipales de la ville autonome de Buenos Aires, il faut ajouter les marchés de gros. Les trois principaux (et les plus proches) sont le mercado central, le marché de la Matanza, spécialisés dans la vente de fruits et légumes frais, et la Megaferia de La Salada, au statut largement informel ${ }^{9}$.

8 Cette enquête a donné lieu à un mémoire de master 2 (Brassamin, 2010). Elle s'inscrit dans le cadre plus large de deux projets de recherche : un programme ECOS SUD (2008-2011) porté par Paul Bouffartigue (directeur de recherches au LEST) et Mariana Busso (chercheure CONICET) sur I'informalité et la précarité, à l'origine de la réflexion sur le formel et l'informel dans l'économie urbaine de Buenos Aires ; un programme PICS CNRS/CONICET (2007-2011) porté par Geneviève Cortes (Professeure à I'université de Montpellier III) et Susana Sassone (directrice de recherche CONICET), sur les évolutions des trajectoires migratoires boliviennes à Buenos Aires et dans les métropoles espagnoles. L'enquête, encadrée par Virginie Baby-Collin, a bénéficié du soutien logistique, scientifique et financier de ces deux projets.

9 La périphérie accueille de nombreux autres marchés et espaces de vente de rue, et de nombreux autres marchés de gros, sur lesquels le travail doctoral de Julie le Gall (2011) apporte des connaissances nouvelles. 
Carte 1 : La population bolivienne dans la région métropolitaine de Buenos Aires en 2001

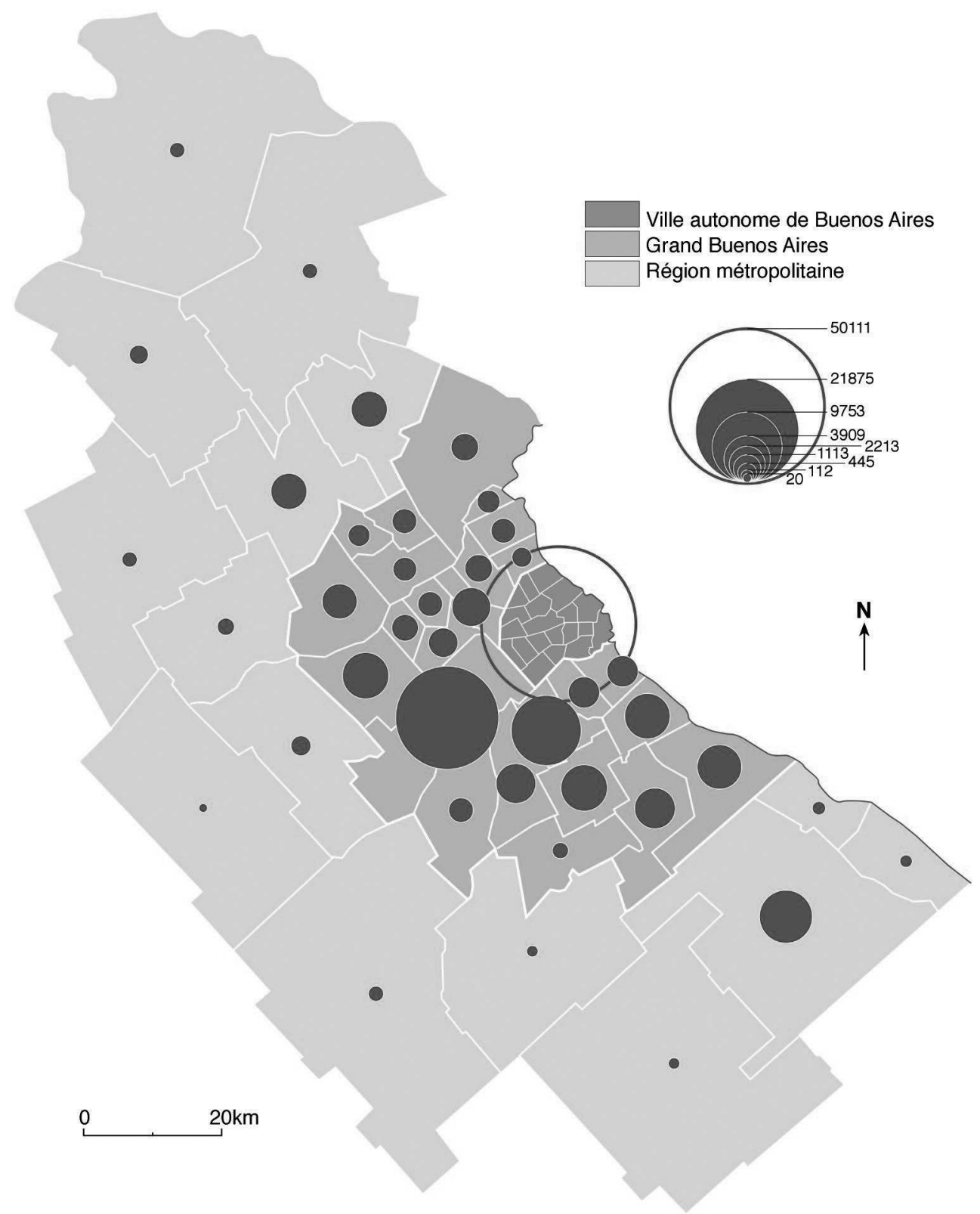

Source : INDEC, recensement 2011. Auteurs : L. Brassamin, V. Baby-Collin et P. Pentsch, 2012. 
Les marchés itinérants auxquels nous nous intéressons ici circulent exclusivement sur le périmètre de la ville autonome de Buenos Aires $\left(200 \mathrm{~km}^{2}\right.$ - contre $3880 \mathrm{~km}^{2}$ pour le Grand Buenos Aires à vingt-quatre municipalités en sus de la capitale - cf. Carte 1) et représentent 161 stands, ce qui équivaut à peine à l'offre d'un grand centre commercial. Peu nombreux, ils n'en constituent pas moins des repères dans le quotidien de la vie commerciale des espaces publics de quartier et une forme spécifique d'encadrement et de contrôle du commerce de rue. Formellement reconnus et gérés par la Direction Générale du Commerce et du Marché (DGCM), ils sont de deux types (FIAB et FIIS - cf. infra). Organisé en tren - train de commerçants fixes (on appartient à tel ou tel) - chaque marché contient un certain nombre de stands enregistrés ${ }^{10}$, vendant des produits regroupés en plusieurs catégories (cf.Tableau 1). Les marchés se tiennent six jours par semaine, doivent respecter des horaires édictés par la DGCM (de 8 heures à 14 heures), ainsi que des localisations précises, conduisant chacun à un itinéraire hebdomadaire de circulation spécifique dans la ville (cf. Carte 2). L'absentéisme des vendeurs est théoriquement sanctionné par les inspecteurs et tout changement de feria est interdit. Les espaces choisis sont généralement des places publiques aérées et verdoyantes, parfois de larges rues, et les règles d'occupation sont strictes : le passage doit être maintenu libre pour les piétons, la circulation automobile ne doit pas être entravée, les extrémités sont bornées. Les rondes quotidiennes des douze inspecteurs de la DGCM veillent au respect de l'occupation de l'espace public et des règles de fonctionnement des marchés (normes sanitaires, d'embauche du personnel, prix fixés chaque semaine par la direction municipale). II existe toutefois, nous le verrons, de nombreuses marges de négociations entre vendeurs et inspecteurs.

Les FIAB appartiennent au patrimoine urbain local. Fondées en 1911 sous le nom de ferias francas, fonctionnant comme marchés de rue municipaux itinérants permettant un approvisionnement à bas prix, elles ont été supprimées pendant la dictature (dans les années 1970) qui les remplaça par des centres d'approvisionnement situés dans des lieux fermés (afin de nettoyer l'espace public), puis sont réapparues avec le retour de la démocratie qui privatise progressivement les centres d'approvisionnement ${ }^{11}$. D'abord informelles, les FIAB ont été I'objet de négociations puis ont obtenu un statut municipal. Sept ferias sont officiellement reconnues dans les années 1990, puis douze en 2001-2002: la crise accroît le commerce informel, et leur reconnaissance est aussi une tentative de contrôle du secteur. Le remplacement du terme de feria franca par celui de FIAB implique leur nouvelle fiscalisation, via leur ré-institutionnalisation. Leur distribution spatiale couvre l'ensemble du territoire municipal, les quartiers bourgeois du nord, comme les zones populaires du sud (cf. Carte 2). Chaque vendeur patenté est installé dans un trailer orange numéroté, camion ou remorque dans lequel les marchandises sont transportées, exposées et vendues, dont les normes de présentation sont homologuées par la municipalité et requises pour obtenir un agrément de vente (mais aux frais de leur propriétaire vendeur, ce qui exige un certain investissement ; cf. Photo 1).

Les FIIS, qui datent du début des années 1990, sont ancrées dans une tradition populaire : elles résultent de la lutte des villeros (habitants) de Villa 20 (quartier populaire de Villa Lugano, cf. Carte 2) pour la reconnaissance puis la formalisation de la vente informelle dans I'espace public. La première FIIS est créée en 1993, avec à l'époque, selon un entretien avec son président, plus de quatre-vingt-cinq stands. La seconde émerge à I'issue de la crise de 2001. La troisième, qui peine à être reconnue (et ne figure pas sur le site Internet de la municipalité), provient d'initiatives informelles dans la zone centrale

10 Les permis sont délivrés par la DGCM. II faut résider dans la municipalité de Buenos Aires pour en bénéficier. Les vendeurs suivent une formation aux règles sanitaires, imposée par la direction. 11 II en reste aujourd'hui sept (centros de abastecimiento), contre plus de soixante-dix dans les années 1980, à la fin de la dictature. 


\section{Photo 1 : FIAB parque Chacabuco}

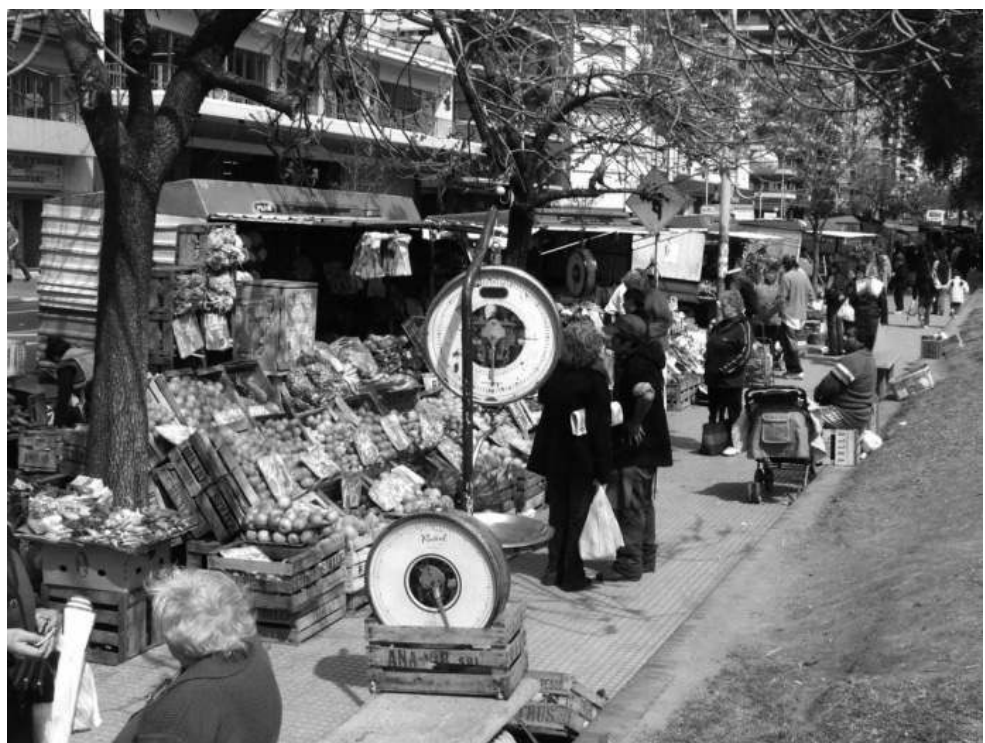

Les différents stands de vente, bien alignés sur le trottoir et en bordure d'un parc, sont reconnaissables au camion orange, obligatoire, labellisé FIAB-Gouvernement de Buenos Aires, qui leur accorde le permis. Ouvert sur le devant, il permet l'étalage des marchandises.

La clientèle est celle des habitués du quartier.

Source : L. Brassamin, 2009.

\section{Photo 2 : FIIS plaza Matheu}

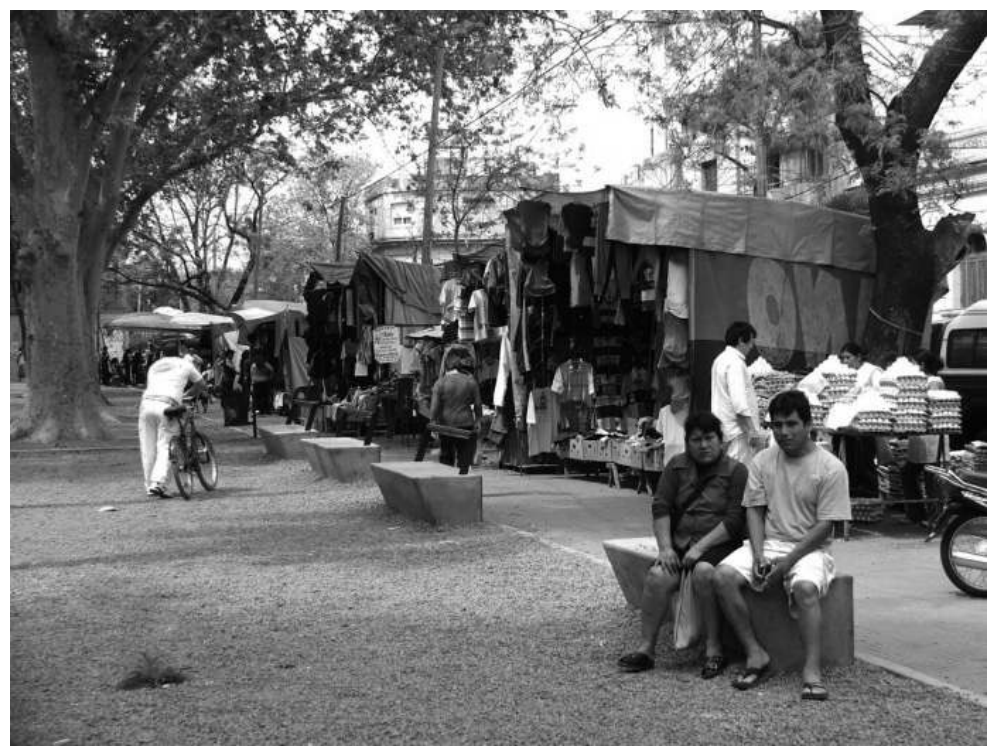

Les stands sont moins homogènes, certains sont bâchés, d'autres non. La vendeuse d'œufs est installée sur de simples tréteaux.

Source : L. Brassamin, 2009. 
Tableau 1 : FIAB et FIIS, quelles différences?

\begin{tabular}{|c|c|c|}
\hline & $\begin{array}{c}\text { FIAB } \\
\text { ferias itinerantes de } \\
\text { abastecimiento barrial, } \\
\text { marchés itinérants de quartier }\end{array}$ & $\begin{array}{c}\text { FIIS } \\
\text { ferias itinerantes de interés } \\
\text { social, plus socialement } \\
\text { orientées }\end{array}$ \\
\hline Nombre de ferias & 12 & 2 (la troisième a été déclassée) \\
\hline Nombre de stands au total & 101 & 60 \\
\hline Nombre de stands par feria & Une douzaine & 30 à 40 \\
\hline Date de fondation & $\begin{array}{c}1911 \text { (ferias francas), puis } \\
\text { années } 1980\end{array}$ & Depuis les années 1990 \\
\hline Couverture géographique & Tout le territoire municipal & $\begin{array}{c}\text { Surtout villas miserias et } \\
\text { quartiers sud }\end{array}$ \\
\hline Types de produits vendus* & $\begin{array}{l}\text { Fruits et légumes, épicerie, } \\
\text { produits fermiers, vêtements, } \\
\text { plantes vertes, produits } \\
\text { ménagers, boulangers }\end{array}$ & $\begin{array}{l}\text { Les mêmes, plus horlogers/ } \\
\text { bijoutiers, cordonniers, } \\
\text { vendeurs de vêtements plus } \\
\text { diversifiés }\end{array}$ \\
\hline Fonctionnement & $\begin{array}{l}\text { Quotidien (six jours par } \\
\text { semaine) }\end{array}$ & $\begin{array}{c}\text { Quotidien (six jours par } \\
\text { semaine) ; I'une d'entre elles } \\
\text { ne fonctionne officiellement } \\
\text { que quatre jours par semaine }\end{array}$ \\
\hline $\begin{array}{l}\text { \% d'étrangers parmi les } \\
\text { titulaires** }\end{array}$ & $24 \%$ & $56 \%$ \\
\hline $\begin{array}{l}\text { \% de femmes parmi les } \\
\text { titulaires }\end{array}$ & $38 \%$ & $59 \%$ \\
\hline
\end{tabular}

* La DGCM accorde des permis de vente différents selon les ferias : on ne peut réparer bijoux ou montres sur une FIAB, c'est possible sur une FIIS. De même, pour éviter la concurrence, le nombre de stands similaires est limité par marché : pas plus de trois stands de fruits et légumes, par exemple.

** Le site internet de la DGCM donne accès au DNI (Document National d'Identité) de chaque titulaire de stand, qui permet d'identifier les étrangers (dont le DNI porte un numéro supérieur à 90000 ).

Source : entretiens DGCM (direction, inspecteurs), observations de terrain et site du gouvernement de Buenos Aires (http://www.buenosaires.gov.ar/areas/espacio_publico/mantenimiento_urbano/ferias_ mercados/ferias_intinerantes.php?menu_id=23759 ; dernière consultation 7 janvier 2012). 
et convoitée de Retiro, quartier résidentiel aisé où se trouve également l'une des plus grandes villas miserias, Villa 31 et 31 bis, proche de la gare de Retiro, point d'entrée à Buenos Aires pour beaucoup de migrants. Les FIIS disposent de plus de stands que les $\mathrm{FIAB}$ (vingt à quarante par feria environ), présentent une plus grande hétérogénéité dans leurs équipements (certains stands ne sont que des planches de bois sur des tréteaux), une plus grande précarité visuelle et des normes moins contraignantes. Légalisées dans le cadre d'une politique d'approvisionnement et d'insertion des villas miserias, elles circulent surtout dans les quartiers populaires du sud de la ville. Les titulaires des stands doivent être résidents des villas miserias pour obtenir une place, ce qui associe étroitement ces marchés à l'économie populaire locale (cf. Carte 2).

\section{Carte 2 : Espaces marchands et population bolivienne dans la ville autonome de Buenos Aires (CABA)}

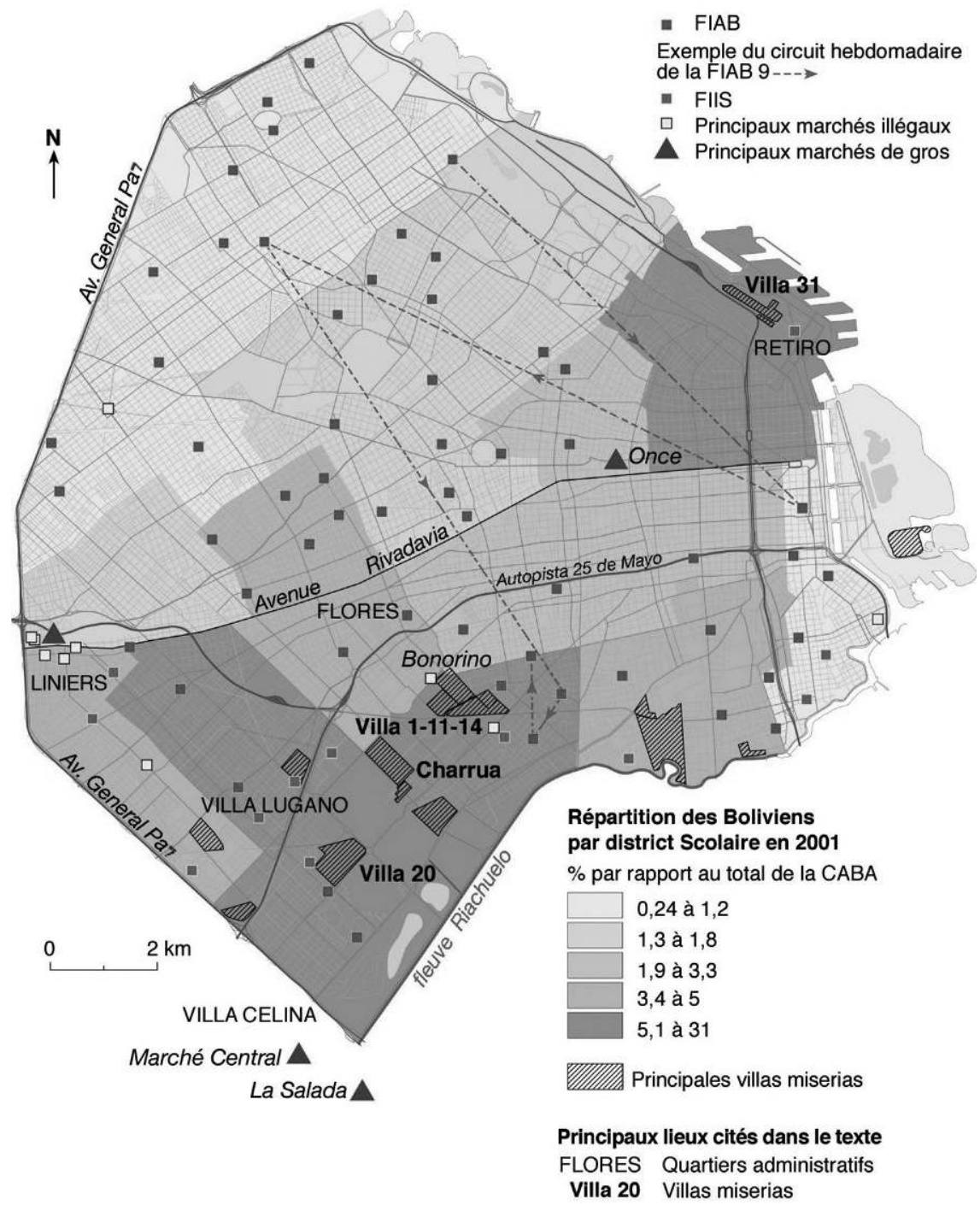

Sources : Gouvernement de Buenos Aires. Observations de terrain, DGCM et INDEC. Auteurs : L. Brassamin, V. Baby-Collin et P. Pentsch, 2012. 


\section{Des marchés pénétrés par des pratiques informelles}

Issus de la formalisation de pratiques commerciales informelles, au début des années 1990 puis 2000, les marchés formels itinérants (FIAB et FIIS) témoignent de croisements permanents entre formalité et informalité commerciale, sur lesquels jouent les titulaires de stands comme les inspecteurs des ferias. La présence des Boliviens y a débuté après qu'ils ont intégré (et contribué à créer) d'autres marchés informels de la capitale (notamment la Feria de la Salada, cf. infra). Sans rentrer ici dans les nombreux débats sur le secteur informel, et considérant une acception large (activités génératrices de revenus, non régulées par l'État ou échappant aux cadres administratifs, fiscaux ou statistiques, dans des contextes sociaux où la réglementation existe - Portes et al., 1989 ; Charmes, 2003), mettons en évidence la souplesse du fonctionnement de ces structures commerciales de rue formalisées, afin de relativiser leur spécificité dans un système commercial de rue où l'informel occupe une large place.

Les pratiques informelles concernent la comptabilité des marchandises, parfois leur provenance (articles de contrefaçon, pour les vêtements notamment), la gestion des employés du stand (non-déclaration des employés), les ajustements sur l'obtention d'un permis (négociation avec le droit pour devenir titulaire). Sur ce dernier point, les entretiens montrent que nombreux sont les titulaires de stand qui ne résident pas dans la municipalité de Buenos Aires, comme requis par la DCGM, mais dans les couronnes suburbaines. La fausse domiciliation est une pratique commune pour obtenir une précieuse autorisation de vente, les places étant limitées en nombre. Sur les dix-sept Boliviens titulaires de stands interrogés sur les FIAB et les FIIS, dix résident en effet hors de la ville autonome de Buenos Aires et ont ainsi rusé pour obtenir leur permis (Tableau 2). Les pratiques clientélistes et l'activation de réseaux relationnels sont un des moyens de négociation : ainsi, bien que la DGCM interdise l'attribution de plusieurs stands au sein d'une même famille, le cas contraire est fréquent chez les Boliviens, attestant de l'efficacité des réseaux familiaux pour contourner la règle, comme de la souplesse des inspecteurs. Lors $d^{\prime}$ 'une visite sur une FIIS avec I'un d'eux, nous observons comment il tolère et protège la présence d'un cordonnier sans permis qui exerce son activité dans un stand bricolé à l'entrée de la feria, en l'assurant du bon avancement de son dossier auprès de la direction du commerce pour obtenir bientôt une légalisation de sa situation. En échange, le cordonnier surveille, à chacune de ses visites, la voiture de l'inspecteur, dans ce quartier situé aux franges d'une villa peu sûre, et lui rend service à l'occasion. Un peu plus loin, un autre vendeur informel qui ne bénéficie pas de cette relation est tout simplement chassé de la place ; il reviendra quand l'inspecteur aura fini sa tournée. Les pratiques clientélistes accordent un pouvoir relativement important aux inspecteurs, auxquels les vendeurs offrent des cadeaux, auprès desquels ils négocient des marges de tolérance à différents niveaux de leur activité : sous-déclaration des produits vendus, pour conserver une marge échappant à la fiscalisation, ou jeu sur les prix (fixés par la municipalité et contrôlés par les inspecteurs) ou encore jeu sur les personnes employées. Outre les quelques vendeurs non accrédités, les employés de stands tenus par des titulaires ne sont pas tous déclarés pour éviter l'acquittement de charges (courant pour les aides familiaux ou migrants sans papiers). Cette embauche informelle permet aussi une certaine flexibilité pour répondre aux variations de charge de travail, notamment les fins de semaine. La forte affluence des week-ends est l'une des raisons du déclassement partiel de l'une des FIIS, qui n'est ces jours-là plus validée par la DCGM, en raison du nombre important de vendeurs non autorisés qui s'y joignent (de 200 à 400, selon un inspecteur, contre quarante stands autorisés !), de l'impossibilité conséquente de contrôler les prix, les normes, et de l'insécurité des environs. Une autre forme d'informalité concerne le non-respect partiel des circuits itinérants établis par la municipalité pour chacune d'entre elles. Lorsqu'un lieu est peu intéressant commercialement, certains vendeurs s'absentent pour tenter une 
journée plus rentable, informellement, ailleurs. Julio, Argentin de parents boliviens, qui gère un stand de fruits et légumes sur une FIAB, et Julieta sa mère, qui y tient un stand d'épicerie, préfèrent ainsi aller vendre les fins de semaine Plaza Saavedra à Belgrano, plutôt que sur la Plaza Echeverria, inscrite sur le parcours officiel de leur FIAB. D'autres vont s'installer sur la gigantesque Feria de Bonorino. L'importance de l'absentéisme sur les FIIS certains jours de semaine révèle aussi la précarité économique des quartiers au sein desquels elles se déploient (cf. Carte 2) et l'écart de revenus qui existe entre FIAB et FIIS, les premières approvisionnant plus fréquemment les quartiers aisés de la capitale.

Ces marchés sont ainsi des espaces de commercialisation où la frontière entre formel et informel est poreuse, où les négociations permettent de diminuer les charges, d'accroître les ventes, etc. Ce système, qui fait appel à la tolérance administrative et aux jeux de clientèle, sait profiter aux vendeurs comme aux inspecteurs. L'informel est ainsi très fonctionnel dans ce système économique et politique (Baby-Collin, 2010 ; Lautier et al., 1991) : perpétuant les fonctionnements clientélistes, sa flexibilité permet à certains d'améliorer leurs revenus (les titulaires), quand d'autres (les employés) y trouvent un travail précaire.

\section{Boliviens et espaces commerciaux de Buenos Aires}

Alors que la présence de vendeurs paraguayens (première nationalité étrangère en Argentine) est dérisoire sur ces marchés, les Boliviens y sont incontournables, se partageant majoritairement les stands avec les Argentins. Les vendeurs étrangers sont majoritaires sur les FIIS, issues des revendications populaires des villas, quartiers informels où les migrants sont souvent dominants, mais les étrangers constituent aussi le quart des titulaires patentés des FIAB (Tableau 1). Si I'on ajoute aux vendeurs patentés les assistants et employés des stands, le pourcentage de population étrangère, essentiellement bolivienne, est encore plus important.

Les Boliviens sont aussi nombreux dans la vente ambulante de rue non réglementée et les grandes ferias informelles (Carte 2). L'une des plus grandes, la Feria de Bonorino, a fait l'objet d'une observation secondaire dans le travail d'enquête. Tous les dimanches dans le quartier de Flores, à proximité d'une des plus grandes villas miserias de la capitale, Villa 1-11-14, elle regroupe quelques 15000 stands, dans un espace densément occupé, aux installations allant du stand-camion aux bâches de plastique tendues entre deux piquets en passant par les produits vendus à même le sol sur une couverture, ou sortis d'un sac à dos et tenus entre les mains. On y trouve de tout, des fruits et légumes frais aux vêtements, en passant par les jouets de contrefaçon, produits ménagers, plats cuisinés (empanadas ${ }^{12}$, asado), et un ensemble de services - coiffeurs, cordonniers, tatoueurs, voyants, etc. Apparu au début des années 1990 à l'initiative de villeros principalement boliviens, ce marché est devenu une des principales megaferias de Buenos Aires, considérée comme incontrôlable par les autorités. La délinquance y est répandue, et les revendeurs d'armes et de drogues profitent de l'affluence pour faire des affaires, situation dénoncée par les marchands qui se font occasionnellement agresser (entretiens avec vendeurs et représentants de l'association des vendeurs, 2009). La DGCM a effectué en 2008 un recensement des 864 membres affiliés à l'association des vendeurs latino-américains de Bonorino, qui a mis en évidence la diversité ethnique des commerçants : Boliviens, mais aussi Argentins, Péruviens, Paraguayens, Chiliens, Uruguayens,

12 Spécialité culinaire de roulé fourré généralement de viande et de légumes. 
Brésiliens, Sierra Léonais, Ghanéens, etc. $98 \%$ d'entre eux habitent dans la municipalité de Buenos Aires ; $88 \%$ d'entre eux n'ont pas de couverture médicale ; $73 \%$ n'ont pas de CUIT ${ }^{13}$.

\section{Photo 3 : Feria de Bonorino}

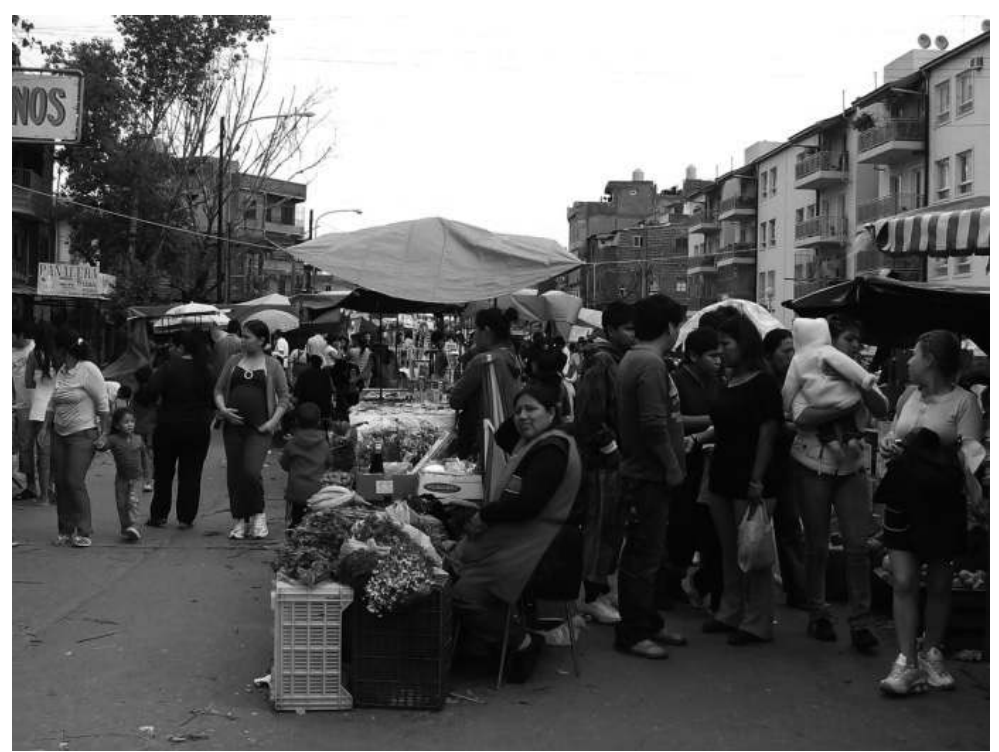

On distingue la variété des types de stands, qui occupent tout l'espace de l'avenue; à gauche les constructions informelles du quartier Rivadavia, à droite les monoblocs de logements sociaux. La foule, un peu clairsemée en ce jour de pluie, est d'ordinaire beaucoup plus dense,

la feria se tenant les fins de semaine.

Source : L. Brassamin, 2009.

La présence bolivienne est importante dans bien d'autres espaces commerciaux de Buenos Aires: dans les centres commerciaux "ethniques " (fréquentés par les Boliviens et affichant des produits d'origine bolivienne) de Liniers et de Nueva Pompeya, où des vendeurs se postent les fins de semaine devant des boutiques fermées alignées les unes à côté des autres ; dans des centres commerciaux classiques et populaires situés dans ces mêmes zones (Cortes, 2001 ; Sassone, 2007) ; sur les marchés de gros des périphéries de l'agglomération, hors du périmètre de la commune autonome de Buenos Aires, qui approvisionnent la capitale en produits frais ${ }^{14}$. Les Boliviens sont aussi à l'origine de la Feria de La Salada, considéré comme le plus grand marché de rue d'Amérique latine et le principal marché noir du pays. La feria a en effet été fondée au début des années 1990 par un groupe de Boliviens, sur des terrains vagues situés de I'autre côté du Riachuelo, à la lisière externe de la ville de Buenos Aires (municipalité Lomas de Zamora). Près de 6000 stands y accueillent 20000 clients venus de toute l'Argentine, voire au-delà, deux fois par semaine, de $1 \mathrm{~h}$ du matin à midi, dans une gigantesque foire bouillonnante (Informe

13 Le CUIT, Code Unique d'Identification Tributaire, est un numéro d'identification de chaque travailleur. Ne pas en posséder signifie que l'on n'est pas inscrit au régime général et que l'on travaille généralement sans être déclaré.

14 Ces espaces sont minutieusement étudiés dans la thèse de Julie Le Gall (2011), qui montre comment le complexe maraîcher des périphéries de la capitale est passé, en trente ans, aux mains des Boliviens, devenus les acteurs essentiels d'un système auparavant contrôlé par d'autres. 
digital metropolitano, 2007). Surtout spécialisé dans le textile et la vente de vêtements (c'est le premier marché national dans ce domaine), on y trouve aussi de l'électronique et des DVD illégalement reproduits et entrés en Argentine via le Paraguay. L'espace est géré par trois associations issues de la partition du premier regroupement de vendeurs des années 1990 qui porte le nom d'Urkupiña (I'une des principales vierges en Bolivie, originaire de Cochabamba) et les Boliviens y restent dominants.

Dans les structures de commerce de rue au détail auxquelles nous nous intéressons ici, les Boliviens, bien plus que toute autre nationalité étrangère, ont acquis une importance croissante depuis une vingtaine d'années. Voyons à présent comment s'est construite cette niche professionnelle, selon quelles trajectoires, au sein de quels réseaux et filières.

\section{Une niche professionnelle bolivienne? Trajectoires de commerçants, réseaux et filières}

Les entretiens, dont le corpus est détaillé dans le tableau 2, ont d'abord ciblé les commerçants boliviens, même si, afin de confronter trajectoires, logiques sociales et économiques, quelques Argentins et d'autres migrants latino-américains (ces derniers étant très minoritaires dans les FIAB et les FIIS) ont été abordés.

Tableau 2 : Corpus des entretiens réalisés auprès des vendeurs sur les ferias lors de l'enquête à Buenos Aires (sept-déc. 2009)

\begin{tabular}{|c|c|c|c|c|c|c|c|c|}
\hline \multicolumn{3}{|c|}{ Espaces de ventes } & \multicolumn{2}{|c|}{ FIAB } & \multicolumn{2}{|c|}{ FIIS } & Bonorino & Total \\
\hline \multirow{2}{*}{\multicolumn{3}{|c|}{ Total entretiens }} & \multicolumn{2}{|c|}{23} & \multicolumn{2}{|c|}{23} & & \multirow{3}{*}{$\begin{array}{c}56 \\
33 \\
(16 ; 17)\end{array}$} \\
\hline & & & \multirow{2}{*}{\multicolumn{2}{|c|}{\begin{tabular}{c|c} 
Titulaires & Employés \\
9 \\
$(5 ; 4)$
\end{tabular}}} & \multirow{2}{*}{\multicolumn{2}{|c|}{\begin{tabular}{c|c} 
Titulaires & Employés \\
15 \\
$(10 ; 5)$
\end{tabular}}} & \multirow{2}{*}{$\begin{array}{c} \\
9 \\
(1 ; 8)\end{array}$} & \\
\hline \multirow{4}{*}{ Boliviens } & \multirow{2}{*}{\multicolumn{2}{|c|}{$\begin{array}{l}\text { Total Boliviens } \\
\text { (hommes ; femmes) }\end{array}$}} & & & & & & \\
\hline & & & 6 & 3 & 11 & 4 & 9 & 33 \\
\hline & $\hat{A} g e$ & \begin{tabular}{|l|}
$20-30$ ans \\
$30-50$ ans \\
$>50$ ans
\end{tabular} & $\begin{array}{l}1 \\
5 \\
0\end{array}$ & $\begin{array}{l}2 \\
0 \\
1\end{array}$ & $\begin{array}{l}0 \\
5 \\
6\end{array}$ & $\begin{array}{l}3 \\
1 \\
0\end{array}$ & $\begin{array}{l}3 \\
4 \\
2\end{array}$ & $\begin{array}{c}9 \\
15 \\
9\end{array}$ \\
\hline & $\begin{array}{l}\text { Durée de } \\
\text { résidence } \\
\text { en } \\
\text { Argentine }\end{array}$ & $\begin{array}{l}<10 \text { ans } \\
10-20 \text { ans } \\
>20 \text { ans }\end{array}$ & $\begin{array}{l}1 \\
3 \\
2\end{array}$ & $\begin{array}{l}1 \\
2 \\
0\end{array}$ & $\begin{array}{l}0 \\
3 \\
8\end{array}$ & $\begin{array}{l}4 \\
0 \\
0\end{array}$ & $\begin{array}{l}2 \\
3 \\
4\end{array}$ & $\begin{array}{l}8 \\
11 \\
14\end{array}$ \\
\hline \multicolumn{3}{|c|}{ Boliviens de $2^{\text {2ème }}$ génération } & 4 & 1 & 1 & 0 & 0 & 6 \\
\hline \multicolumn{3}{|c|}{ Argentins } & 8 & 5 & 0 & 0 & 0 & 13 \\
\hline \multicolumn{3}{|c|}{$\begin{array}{l}\text { Autres nationalités } \\
\text { (Paraguay, Pérou, Uruguay) }\end{array}$} & 0 & 0 & 1 & 2 & 1 & 4 \\
\hline
\end{tabular}

\section{Profils, trajectoires, statuts de vendeurs}

En général, le titulaire d'un stand sur une FIAB, qui circule dans toute la ville, a un statut supérieur à celui d'une FIIS, cantonnée dans les quartiers populaires, lui-même ayant une position plus favorable que le vendeur de feria informelle. Ces situations varient néanmoins beaucoup selon la taille du stand et le type d'activité de vente et il est des Boliviens vendeurs dans la grande feria informelle de La Salada bénéficiant de positions socio-économiques très confortables, tandis que certains stands de FIAB sont peu rentables. Les entretiens éclairent la hiérarchie des stands sur les marchés, l'écart de recettes journalières entre stands pouvant aller de 150 à plus de 8500 pesos par jour (soit un coefficient multiplicateur de plus de cinquante). Les gains les plus faibles sont ceux des stands de produits ménagers, de bazars, ou d'épices (encore que cette catégorie soit 
variable selon les zones), les plus importants étant ceux des stands de fruits et légumes frais, les plus courus, les mieux achalandés et les plus demandeurs de main-d'œuvre ; la viande, les œufs et les produits fermiers se trouvant en situation intermédiaire. II n'est pas rare de compter de six à neuf employés sur un stand de fruits et légumes, une douzaine sur les plus grands, contre trois à quatre sur celui des produits fermiers (volaille, œufs) et rarement plus d'un sur les stands de boulangerie, plantes, épices ou vêtements.

Dans les FIAB comme dans les FIIS, les profils de titulaires et d'employés de stands sont généralement assez différenciés, cette distinction étant peu opérante sur les marchés informels, même si des hiérarchies existent entre vendeurs.

Les employés ont eux-mêmes des statuts variables : de l'aide familial, dont les revenus sont soumis au bon vouloir du patron (voire inexistants), aux employés inégalement déclarés. Les travailleurs les plus précaires sont les employés informels (et plus encore sur les ferias informelles, comme Bonorino) nourris logés chez leur patron, souvent jeunes (moins de trente ans) et migrants récents (arrivés depuis moins de dix ans en Argentine) ; leur salaire est soumis au bon vouloir de leur chef-logeur et ils ne bénéficient d'aucune protection sociale. C'est souvent un emploi considéré comme une première étape ; on espère en sortir en acquérant de l'expérience sur les marchés, pour devenir employé déclaré, quitter le domicile du patron, avoir son propre logement, obtenir une place de titulaire, s'acheter une maison dans un quartier périphérique, ou bien changer de secteur d'activité en trouvant mieux. Sur les stands de fruits et légumes notamment, les employés (ou aides familiaux) résidant au domicile du patron sont nombreux : I'approvisionnement en produits frais nécessite de se lever à $2 \mathrm{~h}$ du matin pour aller chercher les produits sur les marchés de gros, puis les charger sur les camions, avant d'installer le stand sur la feria. À la fin de la journée, il faut recharger le stock, puis l'entreposer dans des locaux qui sont fréquemment au domicile du titulaire. Toutes ces activités requièrent l'usage d'un gros véhicule et leur temporalité nocturne interdit l'utilisation des transports en commun pour se rendre sur les lieux des marchés de gros, ce qui renforce les logiques de cama adentro. L'utilisation de la main-d'œuvre informelle, logée au domicile du patron et corvéable à merci, permet de ne pas avoir à employer de transporteurs (fleteros) ou de porteurs/chargeurs (charangines), les employés s'acquittant de toutes ces tâches en sus de la vente, au prix de très longues journées de travail, aux horaires épuisants (douze heures à seize heures par jour). Cette réduction des intermédiaires, au prix d'un allongement du temps de travail et d'une diversification des tâches, avec des salaires négociables, souvent peu élevés, a notamment été mise en pratique par les propriétaires au moment de la crise.

Les vendeurs indépendants informels connaissent fréquemment une certaine précarité, sur les FIAB comme sur les FIIS (aux marges de la légalité, en négociant avec les inspecteurs). La plupart des commerçants indépendants des marchés informels ont également d'autres lieux de vente en dehors des fins de semaine, dans d'autres ferias ou en marge de marchés officiels, quand la vente dans la feria n'est pas le complément d'une autre activité.

Laura, quarante-huit ans, originaire de la région de Potosi, n'a pas réussi, après seize ans de vie en Argentine, à sortir de la précarité. Ses problèmes de santé et ceux de son mari (ancien mineur de Potosi ayant migré avec elle et leurs six enfants) ont participé à cette stagnation. Elle a successivement exercé les emplois d'aide-cuisinière et de cuisinière sur un marché, dans les années 1990, d'employée domestique (fin des années 1990) et de vendeuse informelle, d'abord à la sauvette dans les rues de la ville, puis, depuis 2008, sur la FIAB X. Deux fois par semaine, elle s'installe aux abords du stand d'Alejandro, titulaire du stand de fruits et légumes, négociant cette tactique de "camouflage " pour vendre quelques racines de gingembre, des citrons, du piment, de l'ail et des épices. 
Trois autres jours de la semaine, elle suit la FIAB Y. Ses revenus ne lui permettent pas de payer les cotisations requises pour régulariser sa situation, mais elle maintient son autonomie en vendant des

produits qui permettent un approvisionnement pas trop fréquent; Alejandro accepte de lui garder son stock et elle se déplace en bus. Même si elle a trouvé une certaine stabilité grâce à la solidarité des vendeurs qui la " protègent ", la précarité de sa situation peut l'amener à plier bagage si un inspecteur un peu trop rigoureux le lui intime.

Les situations comme celles de Laura sont souvent le fait de vendeurs informels ou d'employés précaires âgés de plus de trente ans et présents en Argentine depuis plus de dix ans. Ce sont le plus souvent des femmes, avec charge de famille mais peu de soutiens familiaux et pour qui les contraintes du quotidien ne permettent pas de sortir de la précarité. Leur situation est plus problématique que celle des jeunes employés présentés précédemment, pour lesquels l'emploi précaire et le logement chez le patron sont vécus comme un sas d'insertion, même s'il peut être amené à durer.

Sur les ferias informelles, aux côtés de vendeurs en situation de grande précarité, allant d'un marché à l'autre pour vendre leur marchandise, comme Laura, d'autres complètent des revenus issus soit d'une autre activité, soit de la vente formelle dans $d^{\prime}$ autres espaces le reste de la semaine.

Felicidad, soixante-treize ans, est l'une des plus anciennes vendeuses de Bonorino, considérée comme l'une des fondatrices de la feria en 1991. Arrivée en Argentine depuis La Paz, lorsqu'elle avait neuf ans, elle a d'abord vécu à Jujuy, comme cuisinière et aide-ménagère, avant d'arriver à Buenos Aires à dix-huit ans. Elle a travaillé comme domestique et, passionnée de couture, a pu faire une formation, puis être employée d'une entreprise confectionnant des chemises de marque pour nourrir ses six enfants quand son mari n'avait pas d'emploi. À l'âge de cinquante-cinq ans, elle prend sa retraite, se met à son compte, crée son atelier de couture, embauche à son tour. Vivant dans un appartement de Rivadavia, quartier qui jouxte la Feria de Bonorino, le stand lui a permis d'y vendre ses productions de vêtements pour enfants. Le commerce est une activité secondaire, complémentaire, qu'elle n'exerce que les fins de semaine.

Les profils des titulaires de stands font état de plusieurs types de trajectoires, malgré des caractéristiques communes. Comme le montre le tableau 2, la plupart sont des adultes de plus de trente ans, voire de plus de cinquante ans, résidents en Argentine de longue date (presque tous depuis plus de dix ans, un grand nombre depuis plus de vingt ans). Les titulaires les plus jeunes sont des " héritiers", Boliviens de seconde génération (et donc de nationalité argentine) ayant bénéficié de la situation acquise par leurs parents.

Tel est le cas de Charlie, titulaire d'un stand de produits fermiers sur une FIAB à vingt et un ans, qui a accompagné son père sur les marchés de Buenos Aires depuis l'adolescence ; ce dernier, âgé de quarante-cinq ans, arrivé de Bolivie à l'âge de quinze ans, possède trois stands de produits fermiers, sur deux FIAB (dont l'un est géré par son fils) et une FIIS. II vend aussi le week-end sur une autre feria informelle. Près de trente employés travaillent pour ce commerce, dont cinq en cama adentro, et presque tous au noir. I/ s'agit donc d'une réussite professionnelle que le père de Charlie transmet partiellement à son fils, que l'on peut en ce sens qualifier d'héritier.

Ce cas illustre les liens qui existent entre différents espaces et structures de vente au détail et en particulier les marchés légaux des FIAB/FIIS et les ferias informelles.

De façon générale, les titulaires ont acquis ce statut, relativement favorisé et valorisé, après une trajectoire professionnelle diversifiée (construction, couture, domesticité, services à la personne, etc.), qui a intégré, à un moment donné, la vente de rue sur un stand de marché ou de feria. Pour d'autres, la vente de rue a représenté l'essentiel de leur 
activité professionnelle, et ils y ont progressivement changé de statut pour en gravir les échelons (de vendeur informel à employé, d'employé à titulaire).

Alejandro, trente-huit ans, titulaire d'un stand de fruits et légumes sur la FIAB $n^{\circ} X$ depuis 2002, a travaillé comme employé sur la FIAB dans le stand d'un ami bolivien qui, ayant décidé de rentrer en Bolivie après la crise de 2001, lui a cédé son stand. II a en quelque sorte " hérité " du stand au départ de son compagnon. Chauffeur de taxi à Cochabamba, il avait migré à Buenos Aires à l'âge de vingt et un ans, travaillé dans la construction puis dans le secteur des fruits et légumes, tenant une boutique dans le quartier à forte population bolivienne de Flores, dans le sud de la ville de Buenos Aires, qui a fermé avec la crise de 2001. Propriétaire d'une maison sur deux étages dans le quartier de Villa Celina, près du marché central où il s'approvisionne, il peut y stocker ses marchandises et y

loger ses employés.

Plusieurs éléments peuvent être retenus qui ont favorisé I'accès d'Alejandro au statut de titulaire : une présence ancienne dans le secteur des fruits et légumes, une résidence à Villa Celina, près du marché central d'approvisionnement, et des réseaux relationnels qui lui ont permis de bénéficier d'un stand à l'époque de la crise.

Sur les FIIS, les titulaires (Boliviens en majorité) sont souvent présents depuis l'origine des ferias (dans les années 1990 pour l'une, après la crise de 2001 pour l'autre) : ce sont des migrants de longue date, résidents de Villa 20 ou de Villa Lugano, pour l'une des FIIS, et habitants de Villa Celina, un quartier situé au-delà de la limite municipale de Buenos Aires, à proximité du marché central, pour l'autre. L'ancienneté de la présence des vendeurs témoigne de la faible rotation des titulaires, I'acquisition du statut constituant pour les villeros une certaine forme de réussite sociale. Les titulaires des FIAB s'inscrivent dans des temporalités d'installation plus variées : certains sont là depuis le début des années 1990, mais ils sont plus nombreux à être arrivés au moment de la crise de 2001, qui a vu la création de nouvelles FIAB. Si les profils des Boliviens ou des secondes générations de Boliviens titulaires témoignent soit d'une certaine ascension sociale, soit d'une situation $d^{\prime}$ " héritier ", les entretiens avec les Argentins, pour lesquels existent aussi ces configurations, montrent qu'il existe aussi des " reconvertis " dans l'activité de vente de rue à la suite de la crise. Ainsi un ancien employé d'une compagnie d'assurances, un ancien travailleur de la construction, ou encore un ancien directeur d'école reconverti en 2001 dans la quincaillerie de rue lors de la crise, sont devenus titulaires (I'un d'épices, l'autre de quincaillerie, le dernier de produits ménagers, stands peu consommateurs de maind'œuvre et plutôt peu rémunérateurs). Ces changements de situation sont intervenus suite à une perte d'activité, dans un contexte de croissance de la vente de rue, d'ouverture de nouvelles ferias, et d'opportunités qui ne sont pas liées à des réseaux spécifiques ou une activité commerciale antérieure, mais bien à un contexte de crise.

Les stands de viande sont tenus exclusivement par des Argentins, dans une société fortement consommatrice de produits carnés. En revanche, les Boliviens dominent sur les stands de fruits et légumes, les plus importants des ferias : les cinq stands de fruits et légumes des deux FIIS sont tenus par des Boliviens, dix des dix-neuf stands des FIAB également ; sur les neuf stands restants, la majorité est tenue par des enfants de Boliviens nés en Argentine. Communément, les Argentins et les Boliviens employés, patrons de stands ou inspecteurs, expliquent la présence bolivienne massive sur les marchés par leur forte capacité de travail, leur propension à se " sacrifier " et à travailler seize heures par jour "sans sourciller "(Caggiani, 2005), qui leur aurait permis de gagner leur place dans ce secteur exigeant, délaissé par les Argentins, malgré l'importance des marges qu'il génère. Au-delà de ces interprétations culturalistes, il semble avant tout que se consolident des filières professionnelles boliviennes. 


\section{Niches professionnelles boliviennes sur les marchés}

Le couple migratoire Bolivie-Argentine a plus d'un siècle d'existence et les migrations, auparavant concentrées dans le nord du pays, notamment dans les provinces de Salta et Jujuy près de la frontière bolivienne, se sont dirigées vers la capitale depuis les années 1960. L'ancienneté de cette présence, renouvelée par l'arrivée de nouveaux migrants, permet aujourd'hui d'observer des trajectoires professionnelles ascendantes, inscrites dans le contexte politique et social argentin. Au début des années 1990, quand se formalisent les marchés itinérants et se structurent les foires informelles, les Boliviens s'affirment comme des acteurs majeurs des revendications pour des espaces commerciaux. La conjoncture est celle d'une sortie de crise économique et sociale alors que Carlos Menem applique les plans d'ajustement structurel du FMI pour résorber la dette du pays, quand en Argentine comme ailleurs en Amérique latine les effets de la decada perdida pèsent sur les indicateurs sociaux. Mais la décennie 1990 est néanmoins une période de croissance économique, la parité peso/dollar favorise un développement massif du commerce de contrebande (notamment venu du Paraguay) et la naissance du MERCOSUR (1991-1995) accroît la circulation des biens. Pour les migrants boliviens, de maigres revenus en pesos sont alors de petites fortunes en bolivianos, ce qui participe au maintien d'une forte attractivité de l'Argentine, alors que le commerce informel se structure (réhabilitation des FIAB, création des FIIS, associations de La Salada).

La crise de 2001 accélère considérablement la croissance de l'économie commerciale informelle (boom des Ferias de Bonorino et de La Salada), au sein de laquelle les Boliviens occupaient déjà des positions clés de fondateurs, et permet paradoxalement, pour certains, de consolider leur trajectoire professionnelle, comme en atteste le nombre de migrants devenus titulaires de stands à cette période, qui voit aussi la création de nouveaux postes de titulaires sur des FIAB plus nombreuses, puis la reconnaissance de la seconde FIIS. La faible bancarisation des revenus, caractéristique des migrants, a permis à certains d'amortir mieux que d'autres la crise financière de 2001 et d'investir à bas prix, en liquide, dans un contexte économique déprimé.

Le Gall et Garcia (2008), reprenant la notion d'échelle bolivienne (Benencia, 1997), montrent comment, dans les périphéries maraîchères de Buenos Aires, les Boliviens sont passés de peones (ouvriers journaliers ou saisonniers) à métayers (medieros) puis à producteurs et enfin à commerçants de gros, remontant et contrôlant progressivement toute une filière économique agricole. Le saut de métayer à propriétaire se fait justement durant la crise de 2001, quand les Boliviens prennent le risque d'investir. Leur capacité d'organisation leur a permis de créer de nouveaux marchés de gros dans les périphéries nord et sud de la capitale, qu'ils contrôlent aujourd'hui (leur contrôle sur la production maraîchère de la région est estimé de 25 à $60 \%$, selon les espaces considérés). La dimension générationnelle est ici intéressante : les propriétés agricoles de la région ont longtemps été aux mains des Italiens et des Portugais (que I'on retrouve aussi sur les marchés). Issus de vagues migratoires taries depuis plusieurs décennies, ces migrants sud-européens ont, dans les années 1990 et 2000, progressivement "passé la main " aux Boliviens qu'ils employaient, leurs enfants n'ayant pas repris l'activité familiale. C'est ici que la conjoncture démographique et migratoire, d'une part (des propriétaires vieillissants, de nouveaux acteurs migrants jeunes qui accumulent de l'expérience et du capital) et la conjoncture économique de l'autre, se rejoignent pour expliquer la consolidation de trajectoires boliviennes ascendantes. Enfin, c'est ici aussi que les deux bouts de la filière se rencontrent : d'un côté, des Boliviens qui ont investi le commerce de détail et de rue dans la ville, de l'autre, ceux qui ont investi les périphéries maraîchères et remonté la filière jusqu'aux marchés de gros, des portes de la capitale aux nouveaux marchés des périphéries (Escobar, Lujan, par exemple, au nord). 
Le travail de terrain a permis de mettre en évidence certains liens encore plus étroits, comme sur le stand de fruits et légumes de Julio. La famille est d'origine cochabambine (Julio, la quarantaine, est né en Argentine). Sa mère, doyenne de la FIAB dont elle a participé à la fondation en 1989, a acquis plusieurs stands, elle-même gérant la vente d'épices. Elle a grandi dans les plantations de tabac de Salta, puis vendu des fleurs dans la capitale et acheté avec sa famille, dans des conditions non éclaircies par l'enquête, une quinta dans la périphérie sud de Buenos Aires, à proximité de la Plata, productrice de légumes frais, où travaillent certains des membres de la famille et un certain nombre d'employés. Le stand de Julio vend directement la production de l'exploitation familiale, ce qui est selon lui une clé de son succès : les produits sont de qualité, transportés sans intermédiaires et donc à des prix plus bas, permettant de surcroît des marges satisfaisantes. Les produits de la quinta sont aussi directement commercialisés dans des restaurants de la capitale auxquels Julio va régulièrement livrer la marchandise.

Cette maîtrise des filières commerciales peut se lire aussi dans le secteur des vêtements, bien que les conditions y soient différentes, et que des études restent encore en grande partie à faire à Buenos Aires sur ce sujet. Les ateliers de production textile sont un des secteurs d'activité importants des Boliviens (Montero, 2011) ; souvent employés par des propriétaires d'ateliers coréens, avec des conditions de travail parfois qualifiées d'esclavagistes (Bastia et Mc Grath, 2011), et où l'on retrouve la pratique du cama adentro, les Boliviens sont en position de remontée de filière et de structuration de leurs propres ateliers, comme nous I'avons illustré plus haut avec le cas de Felicidad. On sait les Boliviens dominants au marché de La Salada spécialisé dans I'habillement et les tissus, qu'ils ont contribué à créer, et les entretiens attestent leur présence dans le commerce de détail de vêtements sur les FIAB et les FIIS.

\section{Réseaux, affaires de familles et compadrazgo}

La construction de ces niches professionnelles et le processus de remontée de filières reposent aussi sur le rôle essentiel des réseaux relationnels, communautaires, ethniques, régionaux, de compérage ou de parenté. Leur extension et leur optimisation orientent les trajectoires professionnelles. Dans la tradition andine, le compadrazgo, compérage ou parrainage, joue un rôle fondamental dans l'organisation des liens sociaux. Les nombreux parrains de naissance, de baptême, de mariage, de cérémonies religieuses, inscrivent les individus dans des liens de réciprocité qui sont notamment activés dans la migration. De nombreux entretiens mentionnent le rôle que jouent des compadres, réels ou fantasmés, dans la recherche d'un emploi, pour la régularisation de papiers ou l'obtention d'un logement. Le compérage permet une transmission des savoirs, des connaissances et des réseaux entre les anciens migrants et les nouveaux. Le recrutement des employés sur les stands est majoritairement intra-communautaire et les réseaux sociaux boliviens activés dans le commerce itinérant semblent n'avoir laissé que peu de place à d'autres groupes migrants dans ce secteur.

Les activités commerciales impliquent la famille, surtout dans les stands qui requièrent de la main-d'œuvre, particulièrement les fruits et légumes. La répartition homme/femme des titulaires montre que, si les hommes sont plus nombreux sur les FIAB, plus prestigieuses que les FIIS (et plus rentables en raison de la géographie de leurs espaces de vente), les femmes sont majoritairement titulaires sur les FIIS, ce qui souligne leur place essentielle dans la géographie commerciale locale. Les observations mettent en évidence le rôle central des femmes sur les stands, même quand le titulaire est le mari (on a vu que les stratégies familiales pour occuper plusieurs stands étaient d'utiliser des noms de titulaires différents, puisqu'il est interdit de posséder deux stands). C'est généralement la femme qui tient la caisse dans la journée, glissant un mot gentil aux habitués, mettant un bouquet de persil dans les cabas remplis par les jeunes vendeurs (déclarés ou non). 
Elle surveille et organise le travail des employés. C'est elle aussi qui gère la comptabilité et les achats. Les femmes sont aussi les actrices clés des marchés de gros, allant repérer les produits au petit matin, négocier les achats, préparer les commandes pour le stand (en général deux à trois fois par semaine), que les hommes et les employés viendront plus tard charger et transporter. Le témoignage de David, quarante-quatre ans, employé sur un stand de fruits et légumes et travailleur sur les marchés depuis vingt-deux ans, est éloquent :

"La personne qui gère les stands, l'achat, la vente, l'argent c'est la femme. Elles gèrent tout, elles sont la tête, l'homme non, quoiqu'il gère aussi l'argent. Mais la tête, la colonne vertébrale, c'est la femme, c'est elle qui décide. Ce sont les plus sacrifiées car elles sont les premières à se lever le matin, à 2 h, pour aller choisir la marchandise au marché central, et ensuite elles sont sur les marchés pour la vente. Les hommes y vont pour le transport de la marchandise, mais plus tard, et ils vendent sur le stand".

Le rôle des hommes est plus logistique et politique : ils gèrent notamment le transport des marchandises, entre le marché, le camion et les lieux de stockage (dans les espaces résidentiels souvent). Les enfants sont souvent présents dès leur plus jeune âge, aidant leurs parents après l'école et les fins de semaine, sans être rémunérés. Certains reprennent par la suite le commerce, profitant des réseaux établis, voire obtenant un permis supplémentaire (cf. supra, Julio et Charlie), ce qui témoigne de la transmission intergénérationnelle des savoir-faire et des réseaux, comme la capacité d'extension et d'innovation pouvant favoriser l'ascension socio-professionnelle. Ces héritages ont aussi cours parmi les vendeurs argentins, dont les témoignages évoquent une quatrième génération sur les ferias. Les conditions de travail éprouvantes sur les marchés engagent toutefois à la poursuite d'études afin d'élargir les possibilités d'insertion professionnelle des jeunes vers d'autres secteurs. C'est l'une des raisons du déclin de la présence des vendeurs argentins ou italiens, mais cela reste une stratégie encore rare chez les Boliviens.

\section{De Cochabamba aux villas des périphéries de Buenos Aires : des réseaux et des lieux}

Les trois quarts des commerçants interrogés sur les marchés (titulaires ou employés) sont originaires de la région de Cochabamba, espace pionnier de la migration interne puis internationale en Bolivie (Cortes, 1995 et 2000). Les destinations des Cochabambinos sont surtout urbaines, et leurs emplois orientés vers les services (Baby-Collin, Cortes et Faret, 2009). De son côté, Le Gall (2011) montre que dans les périphéries maraîchères du nord de Buenos Aires les migrants sont plutôt originaires des régions rurales de Potosi ou Tarija, du sud de la Bolivie près de la frontière argentine. Ces observations tendent à confirmer I'hypothèse de filières professionnellement et géographiquement concentrées, aux profils différenciés, qui peuvent se rencontrer à un échelon donné, ici lorsque les grossistes des fruits et légumes rencontrent les détaillants des marchés itinérants. Le fonctionnement des filières joue dans l'activation des relations de compérage et est attesté jusque dans le recrutement de main-d'œuvre bon marché en Bolivie.

Sebastian, vingt-neuf ans, originaire de Cochabamba, est arrivé avec son frère à Buenos Aires en 2003 à la demande d'un compadre, originaire de la même région, qui, ayant émigré depuis longtemps et obtenu un poste de fruits et légumes sur une FIIS, cherchait de la main-d'œuvre pour développer son activité. Six ans après son arrivée, Sebastian est toujours l'employé de son compadre et réside chez lui (cama adentro) à Villa Celina.

Les entretiens évoquent de façon récurrente quelques lieux de résidence privilégiés de Boliviens vendeurs de ferias. Alors que la géographie résidentielle des Boliviens à 
Buenos Aires est complexe (Sassone, 2002 et 2007 ; Cortes, 2001), couvrant de nombreux quartiers et villas miserias de la moitié sud populaire de la ville autonome de Buenos Aires et de nombreux espaces des périphéries (Cartes 1 et 2), la concentration des lieux de résidence des vendeurs interroge sur les liens entre espaces résidentiels et commerçants et leur rôle dans la constitution de la niche professionnelle des vendeurs de ferias.

Tableau 3 : Lieux de résidence des vendeurs boliviens* enquêtés sur les ferias

\begin{tabular}{|l|c|c|c|c|c|}
\hline $\begin{array}{l}\text { Lieu de vente } \\
\text { des enquêtés }\end{array}$ & $\begin{array}{c}\text { Résidents de } \\
\text { Villa Celina }\end{array}$ & $\begin{array}{c}\text { Résidents de } \\
\text { Villa Lugano }\end{array}$ & $\begin{array}{c}\text { Résidents de } \\
\text { Villa 1-11-14 }\end{array}$ & Autres & Total \\
\hline FIAB & 8 & 5 & 0 & 2 & 15 \\
\hline FIIS & 6 & 6 & 1 & 2 & 15 \\
\hline Bonorino & 1 & 0 & 5 & 3 & 9 \\
\hline Total & 15 & 11 & 6 & 7 & 39 \\
\hline
\end{tabular}

* Aux trente-trois vendeurs boliviens interrogés nous ajoutons ici les six Boliviens de seconde génération interrogés (enquête de terrain, 2009).

Villa Celina est un quartier de résidence clé (quinze vendeurs de ferias itinérantes enquêtés sur trente-neuf). Espace de concentration de migrants de Cochabamba, constitué suite à des occupations de terrain informelles dans les années 1980, il bénéficie d'une localisation stratégique, aux portes de Buenos Aires (Carte 2), jouxtant I'un des principaux marchés de gros pour l'approvisionnement en fruits et légumes (le mercado central) et la Feria de la Salada. Nous ne disposons pas d'éléments pour savoir si cette proximité a joué un rôle dans l'origine des marchés (mercado central ou la Salada), mais elle contribue actuellement à la présence importante des Boliviens commerçants. À Villa Celina, un certain nombre de propriétaires de maisons - titulaires de stands - héberge des employés à domicile. Si la mobilité résidentielle des employés logés, ou locataires, peut être forte (et liée à l'emploi), celle des propriétaires dans le quartier est supposée faible - l'acquisition d'une propriété étant un facteur de stabilisation important dans les quartiers populaires (Merklen, 2009) - et les pratiques de logement des employés au sein des maisons des patrons contribuent mécaniquement à accroître la place de Villa Celina comme l'un des espaces privilégiés de résidence des vendeurs.

Villa Lugano, autre pôle résidentiel des commerçants, renvoie à une logique différente : c'est de là qu'est parti le mouvement revendicatif à l'origine de la création des FIIS il y a vingt ans. Le lien originel entre résidents de la villa et présence sur les FIIS s'est maintenu, comme en témoignent plusieurs vendeurs, titulaires depuis les débuts du marché. Le rôle des réseaux relationnels dans l'acquisition des places sur les ferias, comme les formes de sociabilité persistantes des quartiers populaires de villas où l'interconnaissance est grande, ont pu faciliter l'insertion postérieure d'autres résidents locaux (Boliviens ou non) sur les marchés.

Des logiques plus simples de proximité emploi/résidence sont visibles auprès des vendeurs de Bonorino, dont une partie est issue de la villa dans laquelle la feria s'est développée (1-11-14). Les pratiques de vente informelle de proximité sont d'autant plus importantes que l'insécurité locale est forte : I'insertion dans un circuit de vente nécessite un certain nombre de repères et rares sont les vendeurs improvisés. Cette réalité rappelle au passage que l'informalité des ferias s'inscrit dans des régulations fortes qui n'ont rien de spontané et que, dans un monde de mouvement et de réseau, la proximité géographique reste un des éléments de la structuration du quotidien. 
L'hypothèse du rôle central des réseaux dans la structuration des espaces commerciaux boliviens sur les ferias trouve ainsi écho dans les lieux de résidence des vendeurs, à travers quelques quartiers privilégiés, supports de sociabilités et de pratiques ancrées dans des histoires de mobilisation collectives (celles des villas), qui confirment I'hypothèse de canaux localisés favorisant l'accès aux espaces de vente, sur les FIAB et les FIIS tout au moins, issus d'espaces résidentiels investis par les Boliviens, mais activant aussi des formes de recrutement en origine, notamment dans la région de Cochabamba.

\section{Conclusion}

Les marchés itinérants de la ville autonome de Buenos Aires sont révélateurs de dynamiques économiques, socio-professionnelles et spatiales, qui mettent en évidence le fait que des marges informelles et des migrants des milieux populaires surgissent des transformations importantes pour la vie de la cité.

La légalisation progressive des initiatives commerciales informelles témoigne à la fois de la capacité de mobilisation des groupes populaires, pour revendiquer la reconnaissance de leurs pratiques professionnelles, et de la nécessité pour les pouvoirs publics de ne pas se laisser déborder par une informalité galopante, en la régularisant à demi. Les Boliviens s'affirment comme étant des acteurs importants des revendications commerciales. Néanmoins, les jeux constants entre formel et informel sur les marchés, avec la tolérance des inspecteurs (au nombre de douze, pour quatorze ferias à gérer), et plus encore le très grand nombre de ferias informelles restant hors de contrôle, montrent que I'on est encore loin de la généralisation de la formalisation des activités commerciales de rue et que l'informalité est un élément très fonctionnel du système économique présenté : facteur de compétitivité des entreprises commerciales, ou outil d'insertion professionnelle des plus précaires, mais aussi rouage d'un système de pouvoir clientéliste qui perdure. Ainsi, même dans un contexte politique libéral, visant à assainir et contrôler drastiquement l'espace public en le " nettoyant ", comme celui du gouvernement municipal actuel, dirigé par l'homme d'affaires conservateur Mauricio Macri, les dynamiques de chevauchement entre normes et détournement, formel et informel, se maintiennent.

Comme d'autres secteurs de l'économie argentine (celui du maraîchage en particulier), le commerce de rue est l'un des espaces de consolidation d'une niche professionnelle bolivienne à Buenos Aires. Cette situation bénéficie d'une tradition commerciale importante en Bolivie, d'un investissement de l'économie informelle en Argentine marqué par les retombées de la " décennie perdue " au début des années 1990, puis lors de la crise de 2001, qui semble avoir joué un rôle d'accélérateur dans l'intégration des Boliviens à ces activités. Cette dynamique met aussi en évidence certaines spécificités, comme le rôle des économies familiales, I'opérationnalité des réseaux pour consolider la présence dans le secteur, I'efficacité des filières migratoires localisées, et les compétences des Boliviens dans la maîtrise de ces activités commerciales. La diversité des statuts des acteurs de la branche commerciale de détail montre qu'y existent des hiérarchies économiques, des formes d'exploitation forte (chez les employés en cama adentro), mais donne aussi à voir des trajectoires d'ascension professionnelle, en particulier pour les migrants qui ont accumulé réseaux et expériences.

Les espaces de résidence des commerçants des ferias insistent sur la localisation très resserrée des quartiers d'origine des acteurs du commerce itinérant, indicateur supplémentaire de l'extrême localisation des filières professionnelles et migratoires associées aux espaces résidentiels. Ils s'inscrivent dans la géographie des espaces populaires des 
villas miserias du sud de l'avenue Rivadavia ${ }^{15}$, fortement marqués par le fait étranger et le fait bolivien. Mais la présence des Boliviens sur les marchés formels itinérants circulant dans toute la municipalité montre qu'ils sont des acteurs économiques qui ont pris place, au cœur des espaces publics de la cité, aux côtés (voire même à la place) des commerçants argentins, contre balançant par leur rôle croissant une classique géographie de l'exclusion aux marges de la capitale.

\section{Références bibliographiques}

Baby-Collin Virginie (2010) Informalités et inégalités dans les Amériques, in Les Papiers de I'Axe TVME (Territoires, Villes, Modes de vie, Environnement), 6, Toulouse, Presses Universitaires du Mirail, pp. 9-24.

Baby-Collin Virginie, Cortes Geneviève et Faret Laurent (2009) Remises migratoires et développement en Bolivie et au Mexique : une analyse comparée, in Virginie Baby-Collin, Geneviève Cortes, Laurent Faret et Hélène Guétat Bernard Dir., Migrants des Suds. Acteurs, trajectoires et territoires des mobilités internationales, Paris, IRD, pp. 237-260.

Bastia Tanja and Mc Grath Siobhan (2011) Temporality, migration and unfree labour: migrant garment workers, Manchester papers in political economy, Working paper, 6, $38 \mathrm{p}$.

Benencia Roberto (1997) De peones a patrones quinteros. Movilidad social de familias bolivianas en la periferia bonaerense, Estudios Migratorios Latinoamericanos, 12 (35), pp. 63-102.

Brassamin Laurence (2010) Vendeurs boliviens sur les marchés de Buenos Aires, entre formel et informel, trajectoires de migrants sur les marchés itinérants, Aix en Provence, mémoire de Master 2, Sciences Géographiques, Université de Provence, 135 p.

Busso Mariana (2008) Le travail informel, entre théorie et expérience, discussion à partir de I'approche latino-américaine, in Virginie Baby-Collin et Delphine Mercier Dir., Sud à sud, dynamiques sociales et spatiales, Amérique latine/Méditerranée, Aix en Provence, Publications de I'université de Provence, pp. 193-209.

Busso Mariana (2007) Trabajadores informales en Argentina. De la construcción de identidades colectivas a la constitución de organizaciones, Thèse de doctorat de l'université de Buenos Aires (doctorat de sciences sociales) et de l'université de Provence, 402 p.

Caggiano Sergio (2005) Lo que no entra en el crisol. Inmigración boliviana, comunicación intercultural y procesos identitarios, Buenos Aires, Prometeo.

Charmes Jacques (2003). Informel (secteur), in Jacques Levy et Michel Lussault Dir., Dictionnaire de la géographie et de l'espace des sociétés, Paris, Belin, pp. 509-512.

Cortes Geneviève (2008) Migrations, espaces et développement, une lecture des systèmes de mobilité et des constructions territoriales en Amérique latine, Mémoire d'habilitation à diriger des recherches, volume 3, Université de Poitiers.

15 Dans les représentations, cette avenue qui traverse Buenos Aires d'est en ouest limite un nord plutôt bourgeois d'un sud populaire. 
Cortes Geneviève (2001) Les Boliviens à Buenos Aires : présence dans la ville, repères de la ville, Revue européenne des migrations internationales, 17 (3), pp. 119-146

Cortes Geneviève (2000) Partir pour rester. Survie et mutation des sociétés paysannes andines, Paris, ORSTOM, À travers champs, 521 p.

Cortes Geneviève (1995) L'émigration rurale dans les vallées inter-andines de Bolivie, Revue Européenne des Migrations Internationales, 11 (2), pp. 113-128.

Garcia Matias, Le Gall Julie y Mierez L. (2008) Comercialización tradicional de hortalizas de la región metropolitana bonaerense. Herencias, dinámicas e innovaciones de un sistema complejo. Boletín Hortícola de la Facultad de Ciencias Agrarias y Forestales (UNLP), Buenos Aires, 13 (40), pp. 8-15.

Indec (2003) Encuesta complementaria Migraciones Internacionales, ECMI, Buneos Aires, [en ligne]. URL : www.indec.gov.ar

Informe Digital Metropolitano (2007) La Salada, viaje al mercado informal mayorista màs grande de Latinoamérica, 35.

Lautier Bruno, De Miras Claude et Morice Alain (1991) L'État et l'informel, Paris, L'Harmattan, $211 \mathrm{p}$.

Le Gall Julie (2011) Buenos Aires maraîchère, une Buenos Aires bolivienne? Le complexe maraîcher de la Région métropolitaine à l'épreuve de nouveaux acteurs, Thèse de doctorat en géographie, Université Paris I, 776 p.

Merklen Denis (2009) Quartiers populaires, quartiers politiques, Paris, La Dispute, 305 p.

Montero Jeronimo (2011) Neoliberal fashion: The political economy of sweatshops in Europe and Latin America, Thèse de doctorat, Durham University, Durham E-Theses Online, [en ligne] consulté le 11 janvier 2012. URL : http://etheses.dur.ac.uk/3205/

Novick Susana (2008) Migración y políticas en Argentina: tres leyes para un país extenso (1876-2004), in Susana Novick, Las migraciones en America Latina. Politicas, culturas y estrategias, Buenos Aires, CLACSO, pp. 131-152.

Portes Alejandro, Castells Manuel and Benton Lauren (Ed.) (1989) The informal economy. Studies in advanced economies and less developed countries, Baltimore, MD, John Hopkins University Press, 360 p.

Sassone Susana (2007) Migracion, territorio e identidad cultural: construcción de «lugares bolivianos" en la ciudad de Buenos Aires, Población de Buenos Aires, DGEyC, gobierno de Buenos Aires, 4 (6), pp. 9-28.

Sassone Susana (2002) Geografia de la exclusión, inmigración limitrofe indocumentada a la Argentina, del sistema mundo al lugar, Tesis doctoral, Universidad nacional de Cuyo, $742 \mathrm{p}$. 


\section{Laurence Brassamin et Virginie Baby-Collin}

\section{$\cdots$ Prendre place : les Boliviens sur les marchés itinérants de Buenos Aires}

Cet article nuance une classique géographie de l'exclusion des Boliviens étrangers aux marges de la capitale argentine, en analysant, par le biais du commerce de rue itinérant et formalisé, la façon dont les Boliviens ont pris place dans ces espaces commerciaux, et au cœur de la cité, depuis le début des années 1990. À partir d'une enquête de terrain approfondie, il montre comment les Boliviens se sont fait une place dans ces marchés, où les espaces de jeu et de négociation permanente entre formel et informel, tant pour les vendeurs que les inspecteurs, permettent d'accroître les gains des uns, tout en maintenant les pratiques clientélistes de contrôle des autres. Les effets de conjoncture, dans les années 1990 puis lors de la crise de 2001, l'importance des réseaux sociaux et de compérage, les pratiques d'une économie familiale, ont favorisé la constitution de niches professionnelles commerciales boliviennes, non exemptes de hiérarchies sociales, et répondant à des trajectoires migratoires plutôt ascendantes.

\section{... Taking Place: \\ Bolivians in Buenos Aires Itinerant Street Markets}

This article inquiry into the growing importance of Bolivians in Buenos Aires itinerant street markets, since the beginning of the 1990s. It shows how street markets are spaces where sellers as well as work inspectors play a game between formal and informal, which is very functional for both activities, better profits on one hand, clientelist control on the other hand. We explain how conjuncture's effects (in the 90s and after the 2001's crisis), as well as the relevance of the social networks and the habit of familial work, have reinforced the constitution of an ethnic market in Bolivian commercial street activities. This economy is heterogeneous, contains social hierarchies within the Bolivian group, but is also a place for ascendant careers. The Bolivian presence in these street markets circulating over the City of Buenos Aires reveals how they took place in the public space of the city, next to the Argentinian merchants, and how their increasing role moderates the classic geography of their exclusion towards the margins of the capital.

\section{Tomar sitio:}

los bolivianos en las ferias itinerantes de Buenos Aires

Este estudio cuestiona como los bolivianos se insertaron en las ferias itinerantes de Buenos Aires, a partir de los años 1990. Demuestra como las ferias son espacios de juego entre formal e informal, que son funcionales tanto para los vendedores como para los inspectores, permitiendo aumentar las ganancias de unos, y manteniendo practicas clientelista de control de otros. Observamos como los efectos coyunturales (en los 90s y luego en la crisis de 2001), la importancia de las redes sociales y de compadrazgo, las prácticas de una economía familiar, favorecieron la constitución de un nicho profesional comercial boliviano. Este nicho es heterogéneo, observamos hierarquías sociales, pero también trayectorias migratorias frecuentemente ascendentes. La presencia boliviana en esas ferias itinerantes que circulan en la Ciudad Autónoma de Buenos Aires revela como los bolivianos consiguieron afirmar, en el espacio público de la ciudad, al lado de los comerciantes argentinos. Su presencia matiza una geografía clásica de su exclusión hacia los márgenes de la capital. 